\title{
Tracing the Event Evolution of Terror Attacks from On-line News
}

\author{
Christopher C. Yang ${ }^{1}$, Xiaodong Shi ${ }^{1}$, Chih-Ping Wei ${ }^{2}$ \\ ${ }^{1}$ Department of Systems Engineering and Engineering Management \\ The Chinese University of Hong Kong \\ ${ }^{2}$ Institute of Technology Management \\ National Tsing Hua University, Taiwan
}

\begin{abstract}
Since the September $11^{\text {th }}$ terror attack at New York in 2001, the frequency of terror attacks around the world has been increasing and it draws more attention of the public. On January 20 of 2006, CNN reported that al Qaeda leader Osama bin Laden had released a tape claiming that a series of terror attacks were planned in US. These attacks and messages from terrorists are threatening everyone in the world. As an intelligence officer or a citizen in any countries, we are interested in the development of the terror attacks around us. We can easily extract hundreds or thousands of news stories of any terror attack incidents from newswires such as CNN.com but the volume of information is too large for us to capture the information we need. Information retrieval techniques such as Topic Detection and Tracking are able to help us to organize the news stories as events within a topic of terror attack. However, they are incapable to present the complex evolution relationships between the events. We are interested to learn what the major events are in a terror attack but also how they develop within the topic. It is beneficial to identify the starting and ending events, the seminal events and the evolution of these events. In this work, we propose to utilize the temporal relationship, event similarity, temporal proximity and document distributional proximity to identify the event evolution relationships between events in a terror attack incident. An event evolution graph is utilized to present the underlying structure of events for efficient browsing and extracting information. Case study and experiment are presented to illustrate and show the performance of our proposing technique.
\end{abstract}

Keywords: Security informatics, topic detection and tracking, event evolution

\section{Introduction}

Terror attacks are occurring frequently in the recent years and they draw attention of the public. We are threatened and anxious to obtain more information about these incidents. Due to the popularity of the Internet, news stories are now available online at the news information providers' Web sites. We can easily go to any newswires such as CNN, BBC, CBS, etc. or infomediaries such as Google and Yahoo to retrieve news stories for any terror attack incidents in the past. However, the popularity of the online news creates the information overloading problem at the same time. We can 
retrieve hundreds or thousands of news stories for any single terror attack incident without much difficulty; yet it is impossible for users to capture the flow of stories efficiently and effectively from a large volume of news stories.

In the research of topic detection and tracking (TDT), techniques are developed to monitor news stories, spot news events, and track the progress of previously spotted events [4]-[6], [15]-[18]. It clusters news stories as a hierarchical structure as shown in Figure 1. Although users are able to capture the major events in a terror attack incident, it is difficult to capture the development of events within an incident. For example, the out break of a war may evolve to the economic crisis and then evolve to the problem of refugees. In order to capture such evolution relationships, we must consider the temporal information in addition to the content of the news stories.

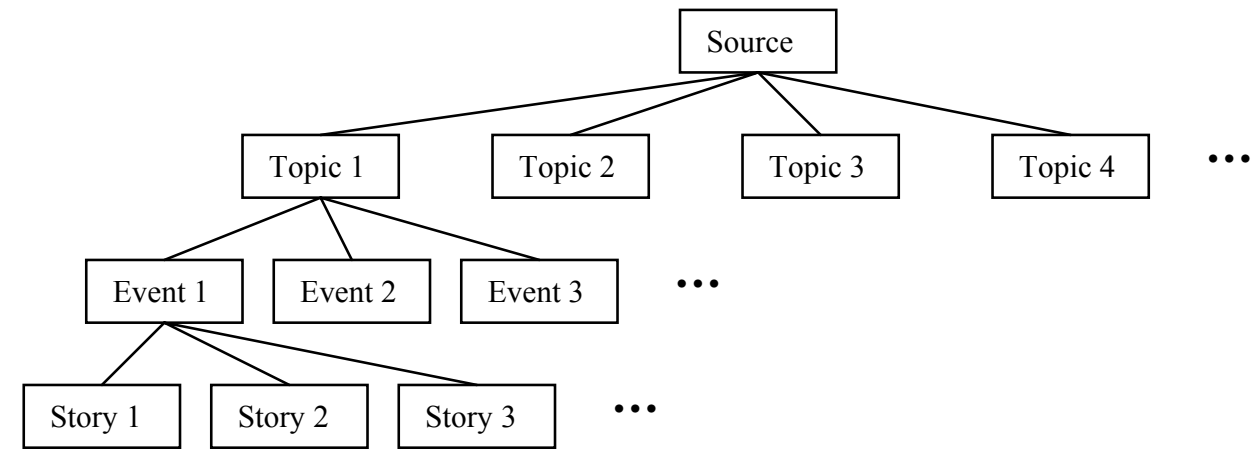

Figure 1 Document organization in topic detection and tracking

In the research of temporal text mining (TTM), techniques are developed to discover temporal patterns in text information over time based on the timestamps of the text streams [11]. In this technique, text steams are partitioned into a number of nonoverlapping sliced time intervals. The theme of each interval is identified and the evolution of theme between successive intervals is extracted. However, the events of the incident are not identified. An event in the terror attack incident may take more than one interval or only part of an interval. The theme extracted from an interval may be part of an event or a combination of several events that occur in the interval. Such technique is not ideal for users to capture the flow of major events in a terror attack incident.

Event evolution is a new concept developed recently. Makkonen [10] was the first to conduct investigation on event evolution as a subtopic of TDT. The news documents within a topic are temporally linearly ordered. A narrative begins when the first story of the topic is detected. A seminal event may lead to several other events. The events at the beginning may have more influence on the events coming immediately after than the events at the later time. As we go through the event in the temporal order, we may see the evolution of events within a terror attack incident. The events and the event evolution relationship can be represented as a graph structure (Figure 2). Nallapati et al. [12] have investigated the dependencies among events and developed a few simple models to determine the event threading relationships from a small number of documents. However, the existing model is rather too simple to capture 
the complex relationships among the events in a large collection of documents within a topic. The concept of event evolution has not been well defined. Wei and Chang [13] proposed an event evolution pattern discovery technique that identifies event episodes together with their temporal relationships that occur frequently in a collection of events of the same type. Their work differs from prior studies in that they focus on segmenting a sequence of news stories of a specific event into event episodes and generalizing event episodes across different events of similar topics. However, relationships among event episodes discussed only consist of temporal ones. In this work, we formally define the event evolution by three logical rules. Besides, we introduce the temporal relationship, event similarity, temporal proximity and document distributional proximity to identify the event evolution relationships to construct the event evolution graphs. Given such graphical representation of the underlying structure of events in a terror attack incident, users can easily navigate the development of the incident and extract specific information for their needs.

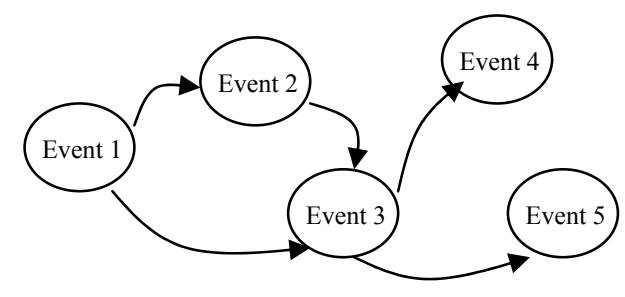

Figure 2. Event evolution graph.

\section{Event Evolution}

Event evolution describes the relationship between two events in a news topic. In order to have a better understanding of event evolution, we must first understand the definitions of story, event, and topic as defined in the research area of topic detection and tracking (TDT). A story is a news article delivering some information to users. An event is something that happens at some specific time but may or may not at a specific place. Although event is defined with a specific place in typical definition in TDT, some events do not have a specific place practically. An event may happen at many places at the same time. A topic is a set of events that are strongly interconnected with each other.

Event evolution is a new concept developed by Makkonen [10], Nallapati et al. [12], and Wei and Chang [13] recently. Makkonen described event evolution as the changing nature of a topic. Nallapati el al. described event threading as the dependencies between events, while Wei and Chang viewed event evolution as temporal dependencies between episodes (i.e., stages or subevents) with an event. We formally define event evolution as a relationship between the events within a topic such that the relationships are narrating the changes of events from the seminal events to the terminal events along the timeline. Such relationship is known as event evolution relationship. We define that the event evolution relationship between event 
A and event B must follow 3 rules if event A evolves to event B: (1) event A must temporally precede event $\mathrm{B},(2)$ event $\mathrm{A}$ must be the necessary and/or the sufficient condition of event $\mathrm{B},(3)$ the event evolution relationship must coincide with the user information needs.

\subsection{Event Evolution Graphs}

To represent the changes of nature within a terror attack incident, we use an event evolution graph to represent the underlying structure of the events and their relationships. Given such event evolution graphs, we can easily identify the seminal events of the terror attack incident and the evolution from the seminal events to other events. For example, the terrorists seizing the Beslan school is the starting event of the "Beslan School Hostage Crisis". The starting event evolves to the event of the attack of Russian special task force. Such Russian attack event is the seminal event that causes the death of the hostages, the investigation of the suspects, the counterterrorism responses from other countries and some other events.

An event evolution graph is a directed acyclic graph (DAG) consisting of events as the nodes and event evolution relationships as the directed edges between nodes. Given a set of $n$ distinct news stories $\mathbf{S}=\left\{s_{1}, s_{2}, \cdots, s_{n}\right\}$ on a given news topic, we have a set of $m$ events $\mathbf{E}=\left\{e_{1}, e_{2}, \cdots, e_{m}\right\}$ and their event timestamps $\mathbf{T}=\left\{t_{1}, t_{2}, \cdots, t_{m}\right\}$. $t_{i}=\tau\left(e_{i}\right)$. Each story is assigned to one of the $m$ events. A directed edge from vertex $e_{i}$ to $e_{j}$ is created in the event evolution graph if there is an event evolution relationship from $e_{i}$ to $e_{j}$. Event $e_{i}$ is the parent of event $e_{j}$ and event $e_{j}$ is the child of event $e_{i} . \mathbf{L}$ is the set of event evolution relationships, $\mathbf{L}=\left\{\left(e_{i}, e_{j}\right)\right.$ where $e_{i}, e_{j} \in \mathbf{E}$. Therefore, the event evolution graph $\mathbf{G}$ is a directed acyclic graph, $\mathbf{G}=\{\mathbf{E}, \mathbf{L}\}$. The most important task in constructing the event evolution graph is identifying the event evolution relationships from the events of the terror attack incident.

Table 1 Eight temporal relationships that satisfy the first rule of event evolution relationship

\begin{tabular}{|c|c|c|}
\hline & Temporal Relationships & Illustration \\
\hline 1 & $t_{A}<t_{B}\left(t_{A}\right.$ before $\left.t_{B}\right)$ & $\begin{array}{llll}\boldsymbol{s}_{\boldsymbol{A}} & \boldsymbol{e}_{A} & s_{B} & e_{B} \\
\end{array}$ \\
\hline 2 & $t_{A} m t_{B}\left(t_{A}\right.$ meets $\left.t_{B}\right)$ & $\begin{array}{lll}\boldsymbol{s}_{\boldsymbol{A}} & \boldsymbol{e}_{\boldsymbol{A}} & \\
& S_{B} & e_{B}\end{array}$ \\
\hline 3 & $t_{A} o t_{B}\left(t_{A}\right.$ overlaps $\left.t_{B}\right)$ & \begin{tabular}{lll}
$\boldsymbol{s}_{\boldsymbol{A}}$ & \multicolumn{2}{c}{$\boldsymbol{e}_{\boldsymbol{A}}$} \\
& $S_{B}$ & $e_{B}$ \\
\end{tabular} \\
\hline 4 & $t_{A}$ di $t_{B}\left(t_{A}\right.$ contains $\left.t_{B}\right)$ & $\begin{array}{lll}\boldsymbol{s}_{\boldsymbol{A}} & & \boldsymbol{e}_{\boldsymbol{A}} \\
S_{B} & e_{B} & \\
\end{array}$ \\
\hline 5 & $t_{A} s t_{B}\left(t_{A}\right.$ starts $\left.t_{B}\right)$ & $\begin{array}{ll}s_{\boldsymbol{A}} & \boldsymbol{e}_{\boldsymbol{A}} \\
S_{B} & \\
\end{array}$ \\
\hline 6 & $t_{A}$ si $t_{B}\left(t_{A}\right.$ started by $\left.t_{B}\right)$ & $\begin{array}{lll}s_{\boldsymbol{A}} & & \boldsymbol{e}_{\boldsymbol{A}} \\
S_{B} & e_{B} & \\
\end{array}$ \\
\hline 7 & $t_{A}$ fi $t_{B}\left(t_{A}\right.$ finished by $\left.t_{B}\right)$ & $\begin{array}{lll}\boldsymbol{s}_{\boldsymbol{A}} & & \boldsymbol{e}_{\boldsymbol{A}} \\
& S_{B} & e_{B} \\
\end{array}$ \\
\hline 8 & $t_{A}=t_{B}\left(t_{A}\right.$ equal to $\left.t_{B}\right)$ & $\begin{array}{ll}\boldsymbol{s}_{\boldsymbol{A}} & \boldsymbol{e}_{\boldsymbol{A}} \\
S_{B} & e_{B} \\
\end{array}$ \\
\hline
\end{tabular}

In order to satisfy the first rule of event evolution relationship that event A must temporally precede event $\mathrm{B}$, we adopt eight temporal relationships among the thirteen temporal relationships as defined by Allen [8]. An event has a timestamp $t$ which is a 
time interval. Given two events, event $\mathrm{A}$ and event $\mathrm{B}$, and their timestamps, $t_{A}=$ $\left[s_{A}, e_{A}\right]$ and $t_{B}=\left[s_{B}, e_{B}\right]$, the eight temporal relationships between event $\mathrm{A}$ and event $\mathrm{B}$ that satisfy the first rule of event evolution relationship are illustrated in Table 1. The distance between $t_{A}$ and $t_{B}, d\left(t_{A}, t_{B}\right)$, is measured as $e_{A}-s_{B}$ if $e_{A}$ is before or equal to $s_{B}$; otherwise $d\left(t_{A}, t_{B}\right)$ equals to 0 .

We utilize the event similarity to identify the pairs of events that satisfy the second and third rules of event evolution relationships. The events that follow the second and third rules share some common information in their content, such as keywords, person and location names. Stories in the pair of events which possess the event evolution relationship usually discuss closely related matters. The authors of the news stories tend to refer the parent event when they are writing the stories of the child event. In our work, we utilize the cosine similarity of term vectors to represent the relatedness of events.

Define a k-term feature space for $\mathbf{S}$ as $\omega=\left\{\omega_{1}, \omega_{2}, \ldots, \omega_{k}\right\}$, then a story $i$ can be represented as a weighting vector $\omega_{i}=\left\{\omega_{i 1}, \omega_{i 2}, \ldots, \omega_{i k}\right\}$. The traditional TF-IDF function is

$$
\omega_{i k}=\frac{t f_{i k}}{\max _{l} t f_{i l}} \log \frac{N}{d f_{k}}
$$

where $t f_{i k}$ is the frequency of term $k$ in document $i . N$ is the total number of documents in that topic and $d f_{k}$ is the number of documents which contains term $k$ in that topic.

We compute the event term vector of event $j$ using the average of the document term vectors of stories that belong to event $j$. We define the event term vector for event $j$ as $\omega_{j}^{\prime}=\left\{\omega_{j 1}^{\prime}, \omega_{j 2}^{\prime}, \ldots, \omega_{j k}^{\prime}\right\}$ where,

$$
\omega_{j k}^{\prime}=\frac{1}{n_{j}} \sum_{\forall s_{i} \in e_{j}} \omega_{i k}
$$

where $n_{j}$ is the number of stories in $\mathbf{S}$ that belongs to event $j$.

The event content similarity is computed as followed.

$$
\cos \_\operatorname{sim}\left(e_{i}, e_{j}\right)=\frac{\sum_{x=1}^{k} \omega_{i x}^{\prime} \omega_{j x}^{\prime}}{\sqrt{\left[\sum_{x=1}^{k}\left(\omega^{\prime}{ }_{i x}\right)^{2}\right]\left[\sum_{x=1}^{k}\left(\omega^{\prime}{ }_{j x}\right)^{2}\right]}}
$$

In addition to the event similarity, we also adopt the temporal proximity and document distributional proximity to measure the strength of event evolution relationships. If two events are distant from each other along the timeline, then the event evolution relationship is less likely to exist between them than those events occur closely with each other. The longer the temporal distance between the two 
events is, the less likely the event evolution between them exists and vice versa. On the other hand, the document distributional proximity supplements the weakness of temporal proximity when there is a burst of number of events in a relatively short period which usually happens at the beginning of the incident. We utilize two decaying functions to reflect the temporal proximity and document distributional proximity.

$$
t p\left(e_{i}, e_{j}\right)=e^{-\alpha\left[\frac{d\left(\tau\left(e_{i}\right), \tau\left(e_{j}\right)\right)}{T}\right]}
$$

where $T$ is the event horizon defined as the temporal distance between the start time of the earliest event timestamp and the end time of the latest event timestamp in the same topic. $\tau\left(e_{i}\right)$ is the timestamp of $e_{i} . \alpha$ is the time decaying factor which is between 0 and 1 .

$$
d f\left(e_{i}, e_{j}\right)=e^{-\beta \frac{m}{N}}
$$

where $m$ is the number of documents that belong to the events happening in-between event $e_{1}$ and $e_{2} . N$ is the total number of documents in the topic. $\beta$ is the document distribution decay factor which is between 0 and 1 .

To measure the strength of the event evolution relationship between two events, we integrate the event similarity function and the two decaying functions to compute the event evolution score as follow:

$$
\operatorname{score}\left(e_{i}, e_{j}\right)= \begin{cases}\cos \_\operatorname{sim}\left(e_{i}, e_{j}\right) \operatorname{tp}\left(e_{i}, e_{j}\right) d f\left(e_{i}, e_{j}\right) & \text { if } \mathrm{e}_{\mathrm{i}} \text { procede } \mathrm{e}_{\mathrm{j}} \\ 0 & \text { otherwise }\end{cases}
$$

In this work, we utilize a static threshold $\lambda(0<\lambda<1)$ on the event evolution scores to identify the event evolution relationships. If the event evolution score of a pair of events is higher than or equal to the static threshold, we consider that there is an event evolution relationship between this pair of events. The event evolution graph is constructed as

$$
\begin{gathered}
\mathbf{G}=\{\mathbf{E}, \mathbf{L}\} \\
\text { where } L=\left\{\left(e_{i}, e_{j}\right) \mid \operatorname{score}\left(e_{i}, e_{j}\right) \geq \lambda\right\}
\end{gathered}
$$

The size of the event evolution graph depends on the granularity of the events. If we decrease the granularity of the events, there will be more number of events. As a result, the complexity of the event evolution graph increases. That means the number of event evolution relationships increase. For example, considering the four events, "Chechen terrorists seized the Beslan school with hostages" (Event A), "Negotiation with terrorists broke down" (Event B), "26 women and infants freed but most hostages were still held" (Event C), and "Special task force assaulted terrorists" (Event D), we may identify the event evolution relationships from Event A to Event B, Event A to Event C, and Event B to Event D. However, if we increase the granularity, we may merge Event A, Event B and Event $\mathrm{C}$ as one single event because these 
events occur in a short period of time and there is only one story in each of the first two events. In this case, the event evolution relationships from Event A to Event B and Event $A$ to Event $C$ are gone but there is one event evolution relationship from the merged event to Event D. The granularity of the event evolution graph should be controlled by users depending on their information needs. Some users may want to have a brief picture of the overall incident at the beginning. Some other users may want to explore more and decrease the granularity when they find interests in the specific events.

\section{Case Study}

In this section, we illustrate the event evolution graph of the terror attack incident. The news stories in our corpus are all extracted from the CNN News website. All stories are written in English. The corpus is generated by automatic crawling and searching with the support of filtering by human annotator. Given the URL of a beginning news story, the crawler analyzed the hyperlinks in the "related stories" section on each page of news story. It eliminated invalid links and crawl the linked news stories. The crawler repeated this process until there were no more links available to analyze or it reached a predefined depth. Since the section of "related stories" is often manually created, it is a good indicator of related stories. Alternatively, the human annotator submitted a query containing topic-specified keywords and obtained the searching results. The system analyzed the hyperlinks in the searching results and crawled related news stories on the submitted topic. Unrelated stories collected in these processes were then filtered out manually.

After the generation of the corpus and filtering of unrelated stories, the human annotator was also instructed to create the truth data of the event evolution graphs. The annotator was first asked to read through the news stories within a specific topic several times and formed a general picture of event evolution graph. In the second step, he was asked to identify the events and assigned each story to one of the events. Given the events, the annotator identified the evolution relationship based on the three rules of event evolution relationships. The annotator reviewed and revised the event evolution graph until no further revision could be made.

In the following subsection, we use the terror attack incident of Chechen Terrorist Seizing Beslan School as an illustration. As discussed in the earlier section, the complexity of event evolution graphs increases as we decrease the granularity of the events. For illustration purpose, we choose a higher granularity in order to present the ideas of event evolutions and the impact of the event evolution scores on the performance of the automatic generated event evolution graphs.

\subsection{Chechen Terrorists Seizing Beslan School}

In the terror attack case of Chechen Terrorist Seizing Beslan School, there are 32 news documents collected from CNN. We have identified 8 events. There are 4 documents per event on average and the maximum and minimum numbers of 
documents per event are 6 and 3, respectively. The details of the events are presented in Table 2.

Table 2. Events in Chechen Terrorists Seizing Beslan School terrorist attach case

\begin{tabular}{|c|c|c|c|c|}
\hline $\begin{array}{l}\text { Event } \\
\text { no. }\end{array}$ & Label & $\begin{array}{l}\text { Number of } \\
\text { doc. }\end{array}$ & $\begin{array}{l}\text { Start } \\
\text { time }\end{array}$ & End time \\
\hline 1 & $\begin{array}{l}\text { Chechen terrorists seized the Beslan school } \\
\text { with hostages, negotiation and some hostages } \\
\text { freed }\end{array}$ & 5 & $\begin{array}{l}2004-09- \\
0201: 46\end{array}$ & $\begin{array}{l}2004-09- \\
0307: 08\end{array}$ \\
\hline 2 & $\begin{array}{l}\text { Special task force assaulted terrorists and } \\
\text { hundreds of hostages were dead }\end{array}$ & 3 & $\begin{array}{l}2004-09- \\
0314: 46\end{array}$ & $\begin{array}{l}2004-09- \\
0505: 14\end{array}$ \\
\hline 3 & $\begin{array}{l}\text { The responses of different parties on the } \\
\text { Beslan school hostage tragic }\end{array}$ & 5 & $\begin{array}{l}2004-09- \\
0415: 45\end{array}$ & $\begin{array}{l}2004-09- \\
07 \text { 13:04 }\end{array}$ \\
\hline 4 & $\begin{array}{l}\text { Russia approached to identify the suspects of } \\
\text { Beslan tragic }\end{array}$ & 6 & $\begin{array}{l}2004-09- \\
0601: 07\end{array}$ & $\begin{array}{l}2004-09- \\
0817: 54\end{array}$ \\
\hline 5 & $\begin{array}{l}\text { Russia conducted investigation and was } \\
\text { determined to put terrorists on trial }\end{array}$ & 4 & $\begin{array}{l}2004-09- \\
0815: 44\end{array}$ & $\begin{array}{l}2004-09- \\
2411: 36\end{array}$ \\
\hline 6 & $\begin{array}{l}\text { Beslan school resumed classes after the } \\
\text { hostage tragic }\end{array}$ & 3 & $\begin{array}{l}2004-09- \\
1408: 12\end{array}$ & $\begin{array}{l}2004-09- \\
1512: 33\end{array}$ \\
\hline 7 & Russia claimed to strike Chechen terrorism & 3 & $\begin{array}{l}2004-09- \\
1408: 52\end{array}$ & $\begin{array}{l}2004-09- \\
1712: 38\end{array}$ \\
\hline 8 & Russia's successive efforts against terrorism & 3 & $\begin{array}{l}2004-09- \\
2912: 01\end{array}$ & $\begin{array}{l}2004-12- \\
1713: 53\end{array}$ \\
\hline
\end{tabular}

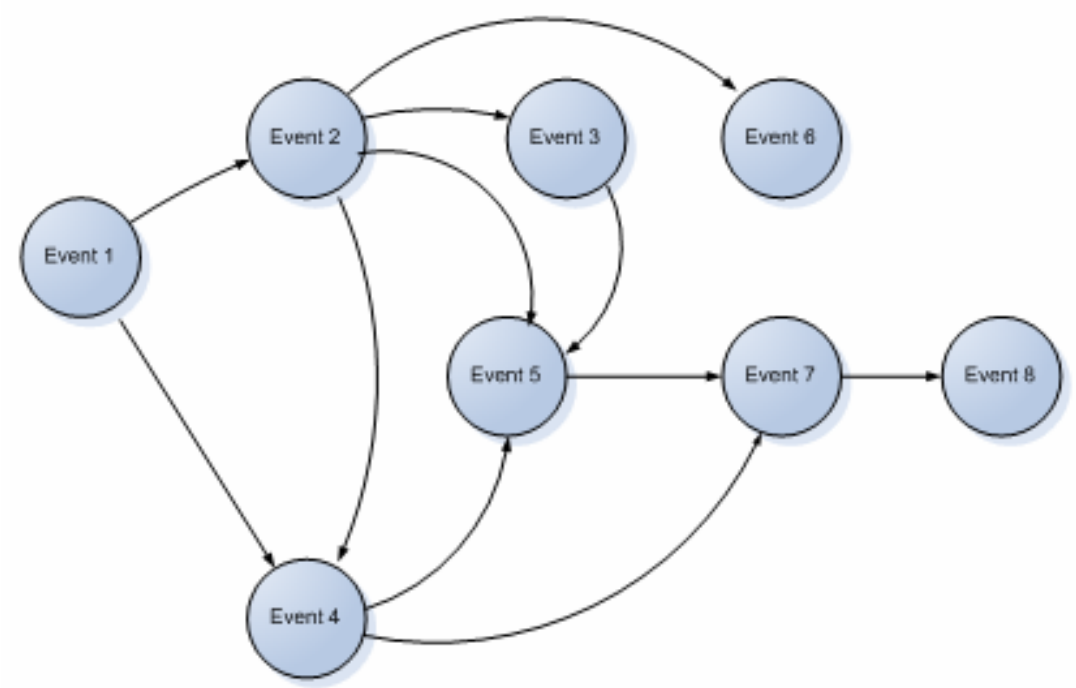

Figure 3. Event evolution graph of Chechen Terrorists Seizing Beslan School terror attack case 
Figure 3 presents the event evolution graph of Chechen Terrorists Seizing Beslan School terror attack case generated by a professional annotator. There are 11 event evolution relationships. Table 3 presents the number of in-links and out-links of the events. Event 2 "Special task force assaulted terrorists and hundreds of hostages were dead" has 4 out-links which is the maximum in the event evolution graph. It can be considered as a seminal event that causes the sequences of events such as the responses of anti-terrorism from different countries, the investigation of the attack and striking the terrorists. Event 6 "Beslan school resumed classes after the hostage" and event 8 "Russia's successive efforts against terrorism" are the terminal events. They can be considered as the final results of the events.

Table 3 Number of in-links and out-links of the events in the Chechen Terrorists Seizing Beslan School terrorist attach case.

\begin{tabular}{|l|c|c|}
\hline Event no. & Number of in-links & Number of out-links \\
\hline 1 & 0 & 2 \\
\hline 2 & 1 & 4 \\
\hline 3 & 1 & 1 \\
\hline 4 & 2 & 2 \\
\hline 5 & 3 & 1 \\
\hline 6 & 1 & 0 \\
\hline 7 & 2 & 1 \\
\hline 8 & 1 & 0 \\
\hline
\end{tabular}

Figure 4 and Table 4 present the result of the event evolution graph generated with the threshold of event evolution score as 0.55 . The precision is 0.73 and the recall is 0.73 .

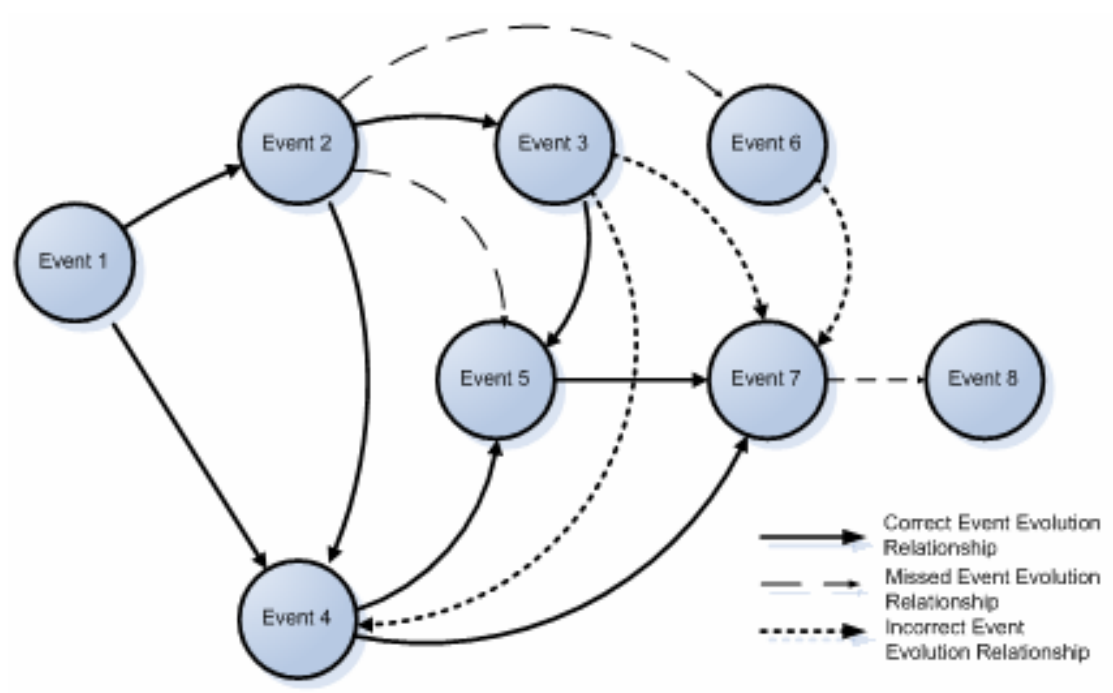

Figure 4. Automatic generated event evolution graph with the threshold of event similarity as 0.55 . 
Table 4. Event evolution relationship in Figure 4

\begin{tabular}{|c|c|c|c|}
\hline $\begin{array}{l}\text { Event Evolution } \\
\text { Relationship }\end{array}$ & $\begin{array}{l}\text { Correct Event } \\
\text { Evolution } \\
\text { Relationship }\end{array}$ & $\begin{array}{l}\text { Missed Event } \\
\text { Evolution } \\
\text { Relationship }\end{array}$ & $\begin{array}{l}\text { Incorrect Event } \\
\text { Evolution } \\
\text { Relationship }\end{array}$ \\
\hline Event $1 \rightarrow$ Event 2 & $\checkmark$ & & \\
\hline Event $2 \rightarrow$ Event 3 & $\checkmark$ & & \\
\hline Event $1 \rightarrow$ Event 4 & $\checkmark$ & & \\
\hline Event $2 \rightarrow$ Event 4 & $\checkmark$ & & \\
\hline Event $2 \rightarrow$ Event 5 & & $\checkmark$ & \\
\hline Event $3 \rightarrow$ Event 5 & $\boldsymbol{\nu}$ & & \\
\hline Event $4 \rightarrow$ Event 5 & $\checkmark$ & & \\
\hline Event $2 \rightarrow$ Event 6 & & $\checkmark$ & \\
\hline Event $4 \rightarrow$ Event 7 & $\checkmark$ & & \\
\hline Event $5 \rightarrow$ Event 7 & $\checkmark$ & & \\
\hline \multirow[t]{4}{*}{ Event $7 \rightarrow$ Event 8} & & $\checkmark$ & \\
\hline & & & Event $3 \rightarrow$ Event 4 \\
\hline & & & Event $3 \rightarrow$ Event 7 \\
\hline & & & Event $6 \rightarrow$ Event 7 \\
\hline
\end{tabular}

Figure 5 and Table 5 present the result of the event evolution graph generated with the threshold of event evolution score as 0.60 . The precision is 0.85 and the recall is 0.55 . When we increase the threshold, we reduce the number of incorrect event evolution relationship; however, we increase the number of missed event evolution relationship. As a result, the precision increases but the recall decreases.

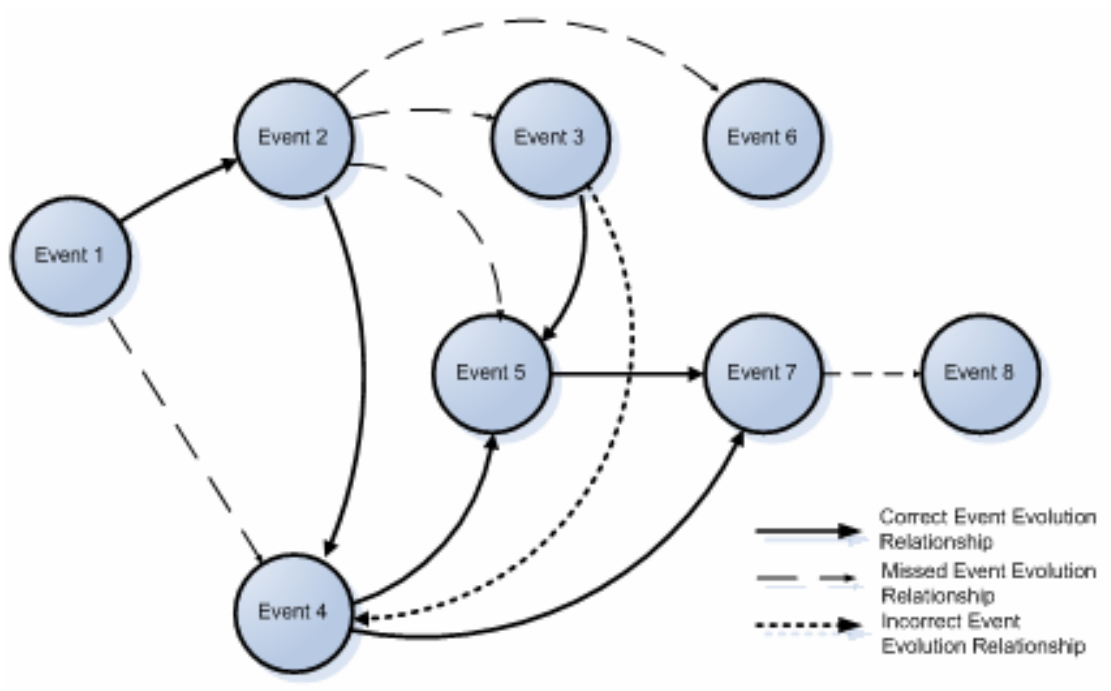

Figure 5. Automatic generated event evolution graph with the threshold of event similarity as 0.60 
Table 5. Event evolution relationship in Figure 5

\begin{tabular}{|c|c|c|c|}
\hline $\begin{array}{l}\text { Event Evolution } \\
\text { Relationship }\end{array}$ & $\begin{array}{l}\text { Correct Event } \\
\text { Evolution } \\
\text { Relationship }\end{array}$ & $\begin{array}{l}\text { Missed Event } \\
\text { Evolution } \\
\text { Relationship }\end{array}$ & $\begin{array}{l}\text { Incorrect Event } \\
\text { Evolution } \\
\text { Relationship }\end{array}$ \\
\hline Event $1 \rightarrow$ Event 2 & $\checkmark$ & & \\
\hline Event $2 \rightarrow$ Event 3 & & $\checkmark$ & \\
\hline Event $1 \rightarrow$ Event 4 & & $\checkmark$ & \\
\hline Event $2 \rightarrow$ Event 4 & $\checkmark$ & & \\
\hline Event $2 \rightarrow$ Event 5 & & $\checkmark$ & \\
\hline Event $3 \rightarrow$ Event 5 & $\nu$ & & \\
\hline Event $4 \rightarrow$ Event 5 & $\checkmark$ & & \\
\hline Event $2 \rightarrow$ Event 6 & & $\checkmark$ & \\
\hline Event $4 \rightarrow$ Event 7 & $\checkmark$ & & \\
\hline Event $5 \rightarrow$ Event 7 & $\checkmark$ & & \\
\hline \multirow[t]{2}{*}{ Event $7 \rightarrow$ Event 8} & & $\checkmark$ & \\
\hline & & & Event $3 \rightarrow$ Event 4 \\
\hline
\end{tabular}

As we observe in the automatic generated event evolution graphs, some event evolution relationships from Event 2 are missed. It is mainly because the temporal distance between Event 2 and the child events are relatively large. The influence of Event 2 to the child events is considered less in our proposed techniques. As a result, they are easily missed. On the other hand, incorrect event evolution relationship from Event 3 to Event 4 is identified by our proposed techniques. It is because there are some overlapping content in the stories of both events and the temporal distance between the two events is small. It is difficult to achieve perfect precision and recall; however, the proposed techniques are promising in producing a meaningful and useful event evolution graph to support user navigation and extraction of information to satisfy their information needs.

\section{Experiment}

We have conducted an experiment to evaluate the performance of our proposed techniques in constructing the event evolution graph. 10 news topics are collected in our corpus. On average, there are 78 news stories per topic, 17.6 events per topic and 4.44 stories per event. From the event evolution graphs we generated manually, there are 24.4 event evolution relationships per topic and 1.4 event evolution relationships per event.

We tested the evolution score function with different parameters:

- CS: Cosine similarity of event term vectors only, without temporal proximity and document distributional proximity. This is equivalent to setting $\alpha=0$ and $\beta=0$ in our suggested event evolution score function; 
- CS*TP: Cosine similarity of event term vectors multiplied by temporal proximity. This is equivalent to setting $\alpha=1$ and $\beta=0$;

- CS*DF: Cosine similarity of event term vectors multiplied by document distributional proximity. This is equivalent to setting $\alpha=0$ and $\beta=1$;

- $C S^{*} \operatorname{sqrt}\left(T P^{*} D F\right)$ : Cosine similarity of event term vectors multiplied by the square root of the product of temporal proximity and document distributional proximity. This is equivalent to setting $\alpha=1 / 2$ and $\beta=1 / 2$.

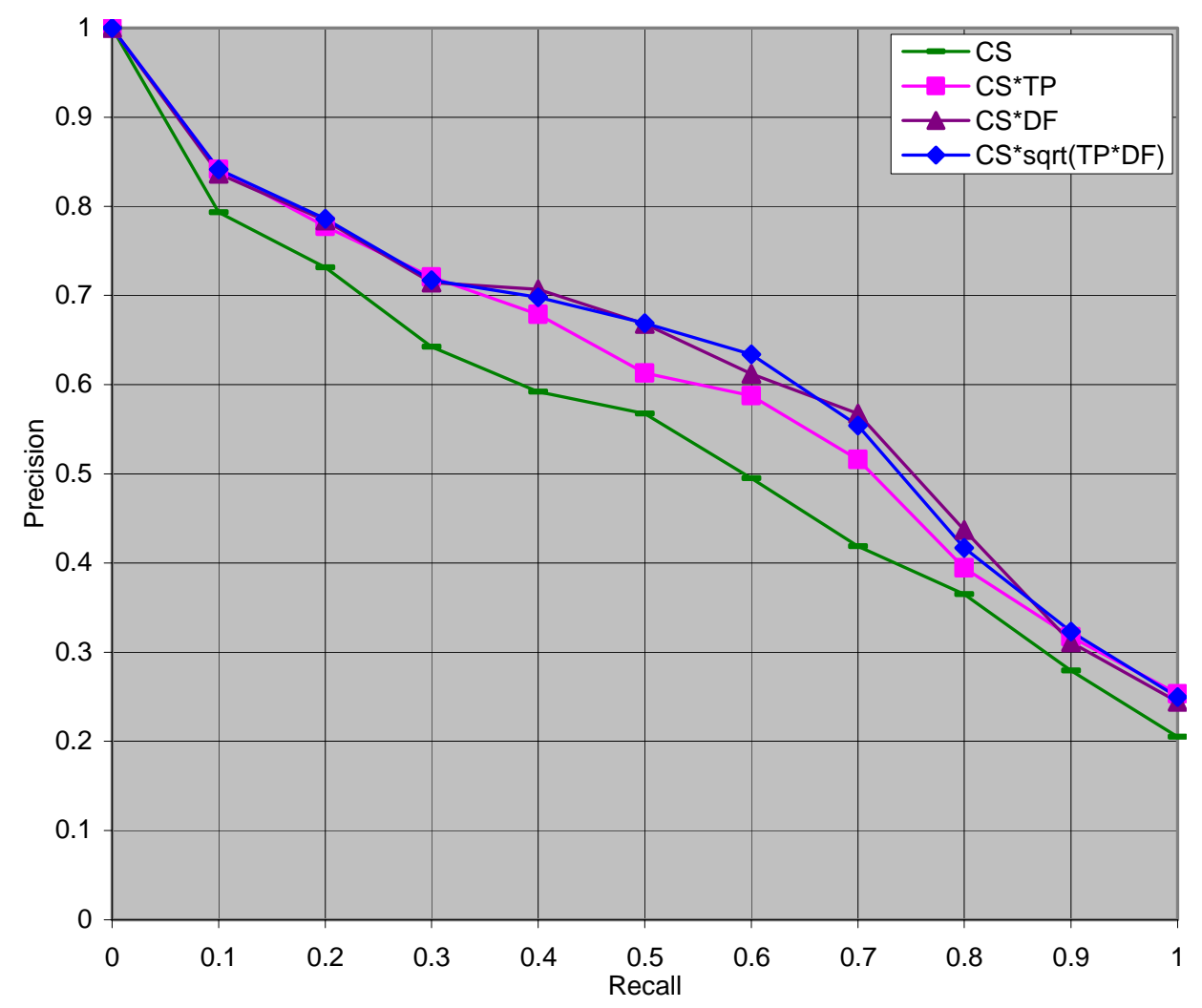

Figure 6 Experimental result

In Figure 6, we observe that both $C S^{*} T P$ approach and $C S^{*} D F$ approach are better than CS approach substantially. This proves that both the temporal proximity and document distributional proximity are helpful in evaluating event evolution relationships. However, it is also interesting to observe that $C S^{*} \operatorname{sqrt}\left(T P^{*} D F\right)$ approach which incorporates both temporal proximity and document distributional proximity does not significantly outperform the $C S^{*} D F$ approach. It may due to the overlapping effect of the temporal proximity and document distributional proximity 
have some overlapping effects. When they are both included, they cancel the effects of each other in some degree.

\section{Conclusion}

There is a large volume of news stories reporting the terror attack incidents on the Web. This information is of great interest to us due to the recent threat of terrorism. In order to capture the development of the events in these terror attack incidents efficiently and effectively, we develop the techniques to identify the event evolution relationships of the events and represent the underlying structure as event evolution graphs. Illustration has been presented. An experiment has been conducted to report the impact of the parameters of the proposed event evolution score function. The performance of the proposed technique is promising. It supports users in efficient navigation of the related events and effectively extracts the specific information that satisfies user information needs. 


\section{References}

1. Aixin Sun and Ee-Peng Lim. Hierarchical Text Classification and Evaluation. In Proceedings of the 2001 IEEE International Conference on Data Mining, pages 521-528. IEEE Computer Society, 2001.

2. Chris Ding, Xiaofeng He. K-means Clustering via Principal Component Analysis. In Proceedings of the 21st International Conference on Machine Learning, Article No. 29, 2004.

3. James Allan. Detection as Multi-Topic Tracking. Information Retrieval, Vol. 5, pp. 139-157, 2002.

4. James Allan, editor. Topic Detection and Tracking: Event Based Information Organization. Kluwer Academic Publishers, 2000.

5. James Allan, Jaime Carbonell, George Doddington, Jonathan Yamron, Yiming Yang. Topic Detection and Tracking Pilot Study Final Report. In Proceedings of the Broadcast News Transcription and Understanding Workshop, 1998.

6. James Allan, Ron Papka, and Victor Lavrenko. On-line New Event Detection and Iracking. In Proceedings of the 21st Annual International ACM SIGIR Conference on Research and Development in Information Retrieval, ACM Press, pp. 37-45, 1998

7. James Allan, Rahul Gupta, and Vikas Khandelwal. Temporal Summaries of New Topics. In Proceedings of the 24th Annual International ACM SIGIR Conference on Research and Development in Information Retrieval, pages 10-18. ACM Press, 2001.

8. James F. Allen. Maintaining Knowledge about Temporal Intervals. In Communications of the ACM, Vol. 26, No. 11, pp. 832-843, 1983.

9. J. Carthy. Lexical Chains for Topic Detection. Technical Report in Department of Computer Science: National University of Dublin, 2002

10. Juha Makkonen. Investigations on Event Evolution in TDT. In Proceedings of HLT-NAACL 2003 Student Workshop, pp. 43-48, 2004.

11. Qiaozhu Mei and Chengxiang Zhai. Discovering Evolutionary Theme Patterns from Text: An Exploration of Temporal Text Mining. In Proceeding of the 11th ACM SIGKDD International Conference on Knowledge Discovery in Data Mining, pp. 198-207, 2005.

12. Ramesh Nallapati, Ao Feng, Fuchun Peng, and James Allan. Event Threading within News Topics. In Proceedings of the 2004 Thirteenth ACM Conference on Information and Knowledge Management, pp. 446-453, 2004.

13. Chih-Ping Wei and Yu-Hsiu Chang. Discovering Event Evolution Patterns from Document Sequences. In IEEE Transactions on Systems, Man, and Cybernetics Part A: Systems and Humans, forthcoming. 
14. Yiming Yang, Tom Pierce, and Jaime Carbonell. A Study on Retrospective and On-Line Event Detection. In Proceedings of the 21st International ACM SIGIR Conference on Research and Development in Information Retrieval, 1998.

15. Yiming Yang, Jaime Carbonell, Ralf Brown, Thomas Pierce, Brian T. Archibald, and Xin Liu. Learning Approaches for Detecting and Tracking News Events. In IEEE Intelligent Systems, Vol. 14, No. 4, pp. 32-43, 1999.

16. Y. Yang, T. Ault, T. Pierce, and C. W. Lattimer. Improving Text Categorization Methods for Event Tracking. In Proceedings of the 23rd International ACM SIGIR Conference on Research and Development in Information Retrieval, Athens, Greece, 2000.

17. Y. Yang, J. Carbonell, R. Brown, J. Lafferty, T. Pierce, and T. Ault. MultiStrategy Learning for TDT. In Topic Detection and Tracking: Event-based Information Organization, J. Allan, Ed. Norvell: Kluwer Academic Publishers, 2002, pp. 85-114.

18. Y. Yang, J. Zhang, J. Carbonell, and C. Jin. Topic-conditioned Novelty Detection. In Proceedings of the 8th ACM SIGKDD International Conference on Knowledge Discovery and Data Mining, 2002. 